\title{
Severe Iron-deficiency Anemia and Celiac Disease
}

\author{
Burz Claudia $^{1,2, *}$, Rares Buiga ${ }^{2}$, Pop Vlad ${ }^{3}$, Laura Urian ${ }^{1,4}$ \\ ${ }^{1}$ Department of Imunology, “Iuliu Hatieganu” University of Medicine and Pharmacy, Cluj-Napoca, Romania \\ ${ }^{2}$ The Oncology Institute "Prof.Dr.I Chiricuta”, Cluj-Napoca, Romania \\ 3“Iuliu Hatieganu” University of Medicine and Pharmacy, Cluj-Napoca, Romania \\ ${ }^{4}$ Department of Hematology, “Iuliu Hatieganu” University of Medicine and Pharmacy, Cluj-Napoca, Romania \\ *Corresponding author: cburz@yahoo.fr
}

\begin{abstract}
We report a case of a 37-year-old woman with atypical presentation of celiac disease in relation to severe iron-deficiency and hyperthyroidism. During the investigation for iron-deficiency anemia, after exclusion of hematologic disorders, she was diagnosed with celiac disease by small-bowel biopsy. After starting the gluten free diet, the serum hemoglobin values were corrected and the symptoms of hyperthyroidism improved. Our presentation demonstrates that for patients with severe iron-deficiency anemia, the examination for the celiac disease should be considered. The quality of life and morbidity of patients with hyperthyroidism, iron-deficiency anemia and celiac disease may be improved by gluten withdrawal.
\end{abstract}

Keywords: anemia, hypertiroidism, screening, celiac disease

Cite This Article: Burz Claudia, Rares Buiga, Pop Vlad, and Laura Urian, "Severe Iron-deficiency Anemia and Celiac Disease.” International Journal of Celiac Disease, vol. 4, no. 1 (2016): 27-29. doi: 10.12691/ijcd-4-1-7.

\section{Introduction}

Gluten enteropathy is an autoimmune disease characterized by permanent intolerance to gliadin and related proteins of cereals (wheat, barley, oats, and rye) that causes an abnormal immune response of the intestinal mucosa [1]. Onset is frequently in childhood, with attenuation or extinction in adolescence and recurrence in adulthood. Subclinical celiac disease can become manifest after surgical aggressions of the gastrointestinal tube. The major symptom is diarrhea associated with weight loss, nonspecific symptoms, nausea, vomiting, bloating, abdominal cramps, and asthenia [2].

Extra intestinal manifestations are the consequence of the malabsorption syndrome: hematological manifestations consist in presence of the anemic syndrome, conditioned by the iron and folic acid absorption, and in the cases with ileal lesions with malabsorption of vitamin B12 occur. Bone, endocrine and neuropsychiatric diseases may be present. Several autoimmune diseases can be associated with celiac disease including autoimmune tyroid disease, type 1 Diabetes Mellitus, multiple sclerosis, autoimmune hepatitis, Addison disease etc. [3,4]. Celiac disease is more common in children and young adults, but in recent years it was observed an increased prevalence in elderly population [5].

The case presented below is of 43 years old patient diagnosed late with celiac disease, respectively at the age of 37, presenting severe anemia.

\section{Case Presentation}

Female patient with hyperthyroidism in medical history, with low compliance to the treatment, presented to the
Hematologic Service with a six-month history of palpitations, asthenia, fatigue, weight loss (approximately $4 \mathrm{~kg}$ in 2 months), dizziness, headache and loss of appetite. Physical examination revealed pale and dry skin and mucous, brittle hair, nails exfoliation (Figure 1), angular cheilitis (Figure 2), without fever, hepatomegaly, signs of dermatitis and arthritis. Blood samples at admission revealed severe microcytic anemia $(\mathrm{Hg}=6.6 \mathrm{~g} / \mathrm{dl})$, MCV 64,3fl, $\mathrm{MCH}=16,1 \mathrm{pg}$. with accelerated sedimentation rate $(\mathrm{ESR}=19-47 \mathrm{~mm})$ and $35 \%$ reticulocytes. Iron studies showed iron-deficiency anemia with hypo sideremia $(\mathrm{Fe}=7 \mu \mathrm{g} / \mathrm{dl})$, low ferritin $($ ferritin $=2.2 \mathrm{ng} /$ $\mathrm{ml}$ ). Red blood cells disarray with microcytic erythrocyte, isolated macrocytes, rare codocytes, rare dacrocytes, rare schizocytes, hypochromia up to anulocytes characterized the medullar examination (Figure 3).

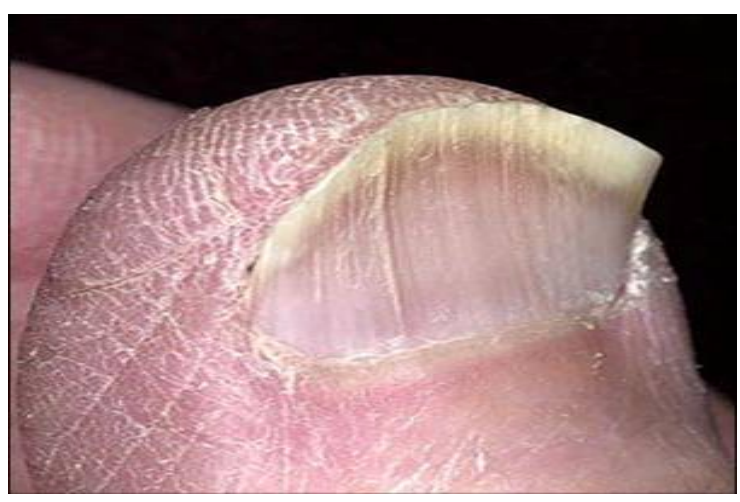

Figure 1. Physical examination of the patient

Considering weight loss, in the absence of digestive symptoms, presence of celiac disease was suspected. The results of serological tests for celiac disease, IgA and IgG anti-transglutaminase antibodies (tTG) measured with ELISA Celikey were positive: IgA $\mathrm{tTG}=>200 \mathrm{U} / \mathrm{ml}$ and 
IgG tTG= $112 \mathrm{U} / \mathrm{ml}$ (normal values $<10 \mathrm{U} / \mathrm{ml}$ ). Later, an upper gastrointestinal endoscopy with biopsy sampling of duodenal mucosa was performed. Histopathological examination confirmed the presence of gluten enteropathy (Figure 4). A positive result for Helicobacter Pylori (HP) was also found. The patient followed a gluten-free diet, iron supplement, antibiotic, antisecretory treatment for the gastritis and HP. Two months after starting her diet without gluten, the patient was no longer anemic $(\mathrm{Hg}=$ $12.9 \mathrm{~g} / \mathrm{dl}, \mathrm{HT}=39,1 \%$ ) and her symptoms improved.

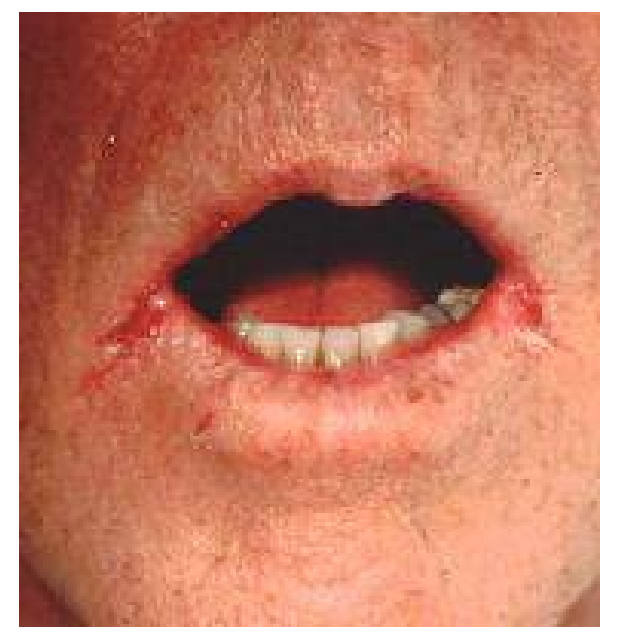

Figure 2. Physical examination of the patient

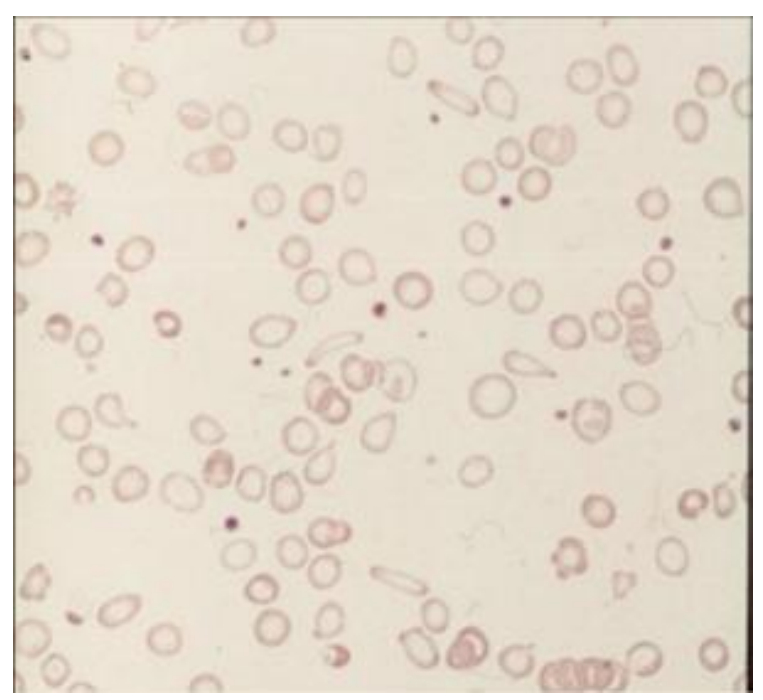

Figure 3. Medullar examination

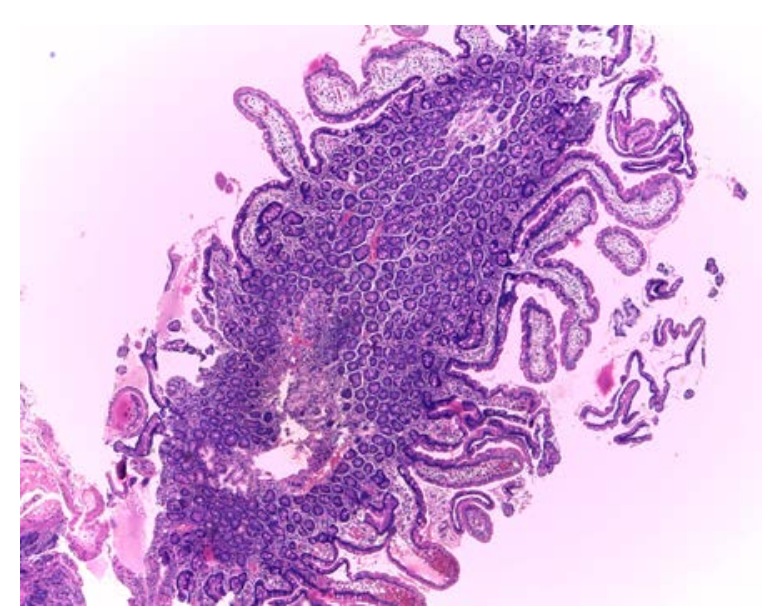

Figure 4. Histopathological examination

\section{Discusions}

Last decades have shown a shifting from early childhood to late childhood age, adult or elderly and the tendency to become gender equalization (formerly predominance of females) of the disease [6]. The presence of celiac disease should be considered in patients presenting severe anemia and hyperthyroidism. Several causative factors for explaining the mechanism of anemia in celiac disease were proposed the most common being abnormal iron absorption secondary to villous atrophy of the intestinal mucosa [7], increased blood loss, correlated with severity of villous atrophy and malabsorption of various micronutrient necessary for normal hematopoiesis. The involvement of some pro-inflammatory cytokine in celiac disease [8] such interferon gamma, interleukin 6 considered powerful mediators of hypoferremia in inflammation by synthesis of the iron regulatory hormone hepcidin are common in anemia of celiac disease.

The diagnosis for the celiac disease is based on antitransglutaminase antibodies and antiendomysial antibodies, test for exclusion of gluten and the morphological criteria (mucosal atrophy) as golden standard, followed by glutenfree diet which is essential for the clinical, biological and histological improvement [9]. In the case of our patient the disease started with extra intestinal manifestation, the anemia, due to the malabsorption of iron and folic acid. Hematologic manifestations are the most common extra intestinal manifestations in celiac disease. If an anemic syndrome is suspected, after the exclusion of other causes, celiac disease may be considered, even in the absence of any gastro intestinal tract symptoms. Our case presented the serological markers positive. Celiac disease diagnosis was confirmed after upper gastrointestinal endoscopy with duodenal mucosal biopsy and histopathological examination.

The efficient treatment of celiac disease remains the withdrawal of gluten from the diet $[10,11]$ which allows the small bowel mucosa to normalize and may correct refractory anemia and hyperthyroidism. The early diagnosis of celiac disease may prevent or reverse extraintestinal symptoms including osteoporosis, anemia, fatigue, and may reverse some thyroid abnormalities [12]. In our case, the anemia of patient significantly improved on a gluten free diet and the symptoms of hyperthyroidism also improved without anti thyroid medication. These finding suggest the possibility to correct the thyroid abnormalities by gluten free diet. Without a correct treatment, several complications such as malabsorption, osteoporosis, infertility and lymphoma may occur.

\section{Conclusions}

Celiac disease can appear at any age, but in adulthood it appears mostly in the mid fifty's. We report a case of a patient with severe anemia, hypertiroidism and celiac disease. In this particular case, the late diagnosis indicates the subclinical manifestation of the disease that defines the presence of the intestinal morphological lesions compatible with gluten enteropathy, but has a minor symptomatology (anemia, osteoporosis). The patient thyroid function improved and anemia normalized after a gluten free diet. 


\section{References}

[1] Rubio-Tapia A1, Hill ID, Kelly CP, Calderwood AH, Murray JA; American College of Gastroenterology. ACG clinical guidelines: diagnosis and management of celiac disease. Am J Gastroenterol. 2013;108(5):656-76; quiz 677.

[2] Sur G, Floca E, Sur L, Sur D, Samasca G. Clinical presentation of celiac disease; Masks therapeutic perspectives of celiac disease. Pharmaceutica Analytica Acta 4 (4):1000228.

[3] Singh P, Makharia GK. Non-classical Celiac Disease: Often Missed. International Journal of Celiac Disease. 2014; 2(3):76-85.

[4] Kumar M, Rutecki GW. Atypical Celiac Disease: Could You Be Missing This Common Problem? Consultant. 2010;50(3):121-128.

[5] Vilppula A, Kaukinen K, Luostarinen L, et al. Increasing prevalence and high incidence of celiac disease in elderly people: a population-based study. BMC Gastroenterol. 2009; 9(1): 49.

[6] Thomson AB. Small intestinal disorders in the elderly. Best Pract. Res. Clin. Gastroenterol. 2009; 23(6): 861-874.
[7] Freeman HG. Anemia in Adult Celiac Disease; Presentation with Iron Deficiency Anemia Masked by Colon Cancer. International Journal of Celiac Disease. 2015;3(1):33-36.

[8] Samasca G, Lupan I, Deleanu D, Cristea V, Makovicky P. Immunological approach of the challenges of the XXI century in celiac disease. International Reviews of Immunology 2014; 33(1): 3-8.

[9] Samasca G, Sur G, Lupan I. Current Trends and Investigative Developments in Celiac Disease. Immunological Investigation 2013; 42(4), 273-284.

[10] Theethira TG, Dennis M. Celiac disease and the gluten-free diet: consequences and recommendations for improvementDig Dis. 2015;33(2):175-182.

[11] Samasca G, Sur G, Lupan I. Deleanu D. Gluten-free diet and quality of life in celiac disease. Gastroenterology and Hepatology from bed to bench 2014;7(3):139-143.

[12] Paul SP, Basude D. Recognition and management of coeliac disease in children. J Fam Health Care. 2013 Dec;23(8):28-30, 32-35. 